eft ventricular (LV) hypertrophy may reflect physiological adaptation to an increased work load of the heart following intense physical training. However, LV hypertrophy often -represents a pathophysiologic condition, and can develop due to intrinsic stimuli (cardiomyopathy), or secondary to extrinsic stimuli, such as pressure or volume overload accompanying hypertension and valvar disease (fig 1). Myocardial hypertrophy is also part of the remodelling process following an acute myocardial infarction, and is a common finding in patients with congestive heart failure caused by systolic and/or diastolic LV dysfunction.

The presence of LV hypertrophy is a strong independent risk factor for future cardiac events and all cause mortality. ${ }^{1}$ The risk for sudden cardiac death is increased in subjects with LV hypertrophy, independent of the aetiology, and this event occurs also in individuals with no or only mild prior symptoms related to cardiovascular disease. Intense animal and human research has identified some common features and provided a framework within which some crucial components can be explained. Thus, whether the focus is haemodynamic or electrophysiologic, there seems to be consensus as to the initiation of a fetal-like gene programme in myocardial hypertrophy. ${ }^{23}$ The insights into its consequences can be applied in the clinical management of individual patients.

During recent years, experts with different backgrounds within cardiovascular medicine have aimed to create a united picture of cardiac hypertrophy and sudden cardiac death, and to present it in such a way that in can be integrated and applied into clinical cardiovascular practice. This article is an attempt to pursue this ambition. We focus on LV hypertrophy in human hypertension as a model of cardiac hypertrophy, and myocardial hypertrophy as a condition with "reduced repolarisation reserve", ${ }^{\prime \prime l}$ and thus a latent acquired long QT syndrome (LQTS). ${ }^{\mathrm{w} 2}$ We will not cover aspects on hypertrophic cardiomyopathy, a topic which has already been addressed in an excellent contribution within this series, ${ }^{\text {w3 }}$ and will not attempt to discuss atrial fibrillation, for which recent excellent guidelines are available, ${ }^{\mathrm{w} 4}$ although there are several important relations between these topics (fig 2).

\title{
DEVELOPMENT OF LV HYPERTROPHY
}

See end of article for authors' affiliations

Correspondence to: Professor Lennart Bergfeldt, Department of Cardiology, Sahlgrenska University Hospital/S, S-413 45 Gothenburg, Sweden; lennart.bergfeldt@hil.gu.se
The myocardium has three morphological compartments: (1) the muscular compartment consisting of myocytes, the dominant cell type of the normal heart comprising $\sim 30 \%$ of the myocardial cells and $\sim 70 \%$ of cardiac tissue volume; (2) the interstitial compartment formed by fibroblasts and collagen; and (3) the vascular compartment with smooth muscle and endothelial cells. An increase in LV wall stress-for example, caused by hypertension induced increase in afterload-will stimulate myocyte hypertrophy, collagen formation and fibroblasts, and thus remodelling of the myocardium with a disproportionate increase in fibrous tissue. These changes will subsequently reduce LV compliance, leading to diastolic dysfunction. Structural changes of the coronary arteries and the increase in both interstitial myocardial fibrosis and in myocardial mass contribute to reduce the vascular coronary flow reserve. In addition, myocardial ischaemic episodes cause transient diastolic dysfunction (figs 3 and 4).

An increase in LV wall stress is the principal mechanical factor in the development of LV hypertrophy, and blood pressure the most powerful determinant of LV mass. However, some additional haemodynamic factors play important roles in the development and maintenance of LV hypertrophy (fig 5). ${ }^{4}$ Thus, volume overload also contributes importantly to the development of cardiac hypertrophy. Although the exact mechanism by which sodium intake influences LV mass is unclear, a high salt intake could expand intravascular volume and increase LV preload. Hypertrophy of the arterial resistance vessels with an increased peripheral vascular resistance is present in established hypertension. ${ }^{\text {w5 }}$ Also the carotid arteries and other large arteries show structural changes. These structural vascular changes with increased arterial stiffness lead to enhanced reflection of the arterial pulse wave, and the resulting increase in systolic blood pressure may promote the development of LV hypertrophy. ${ }^{\mathrm{w} 6}$ While diastolic blood pressure is more 


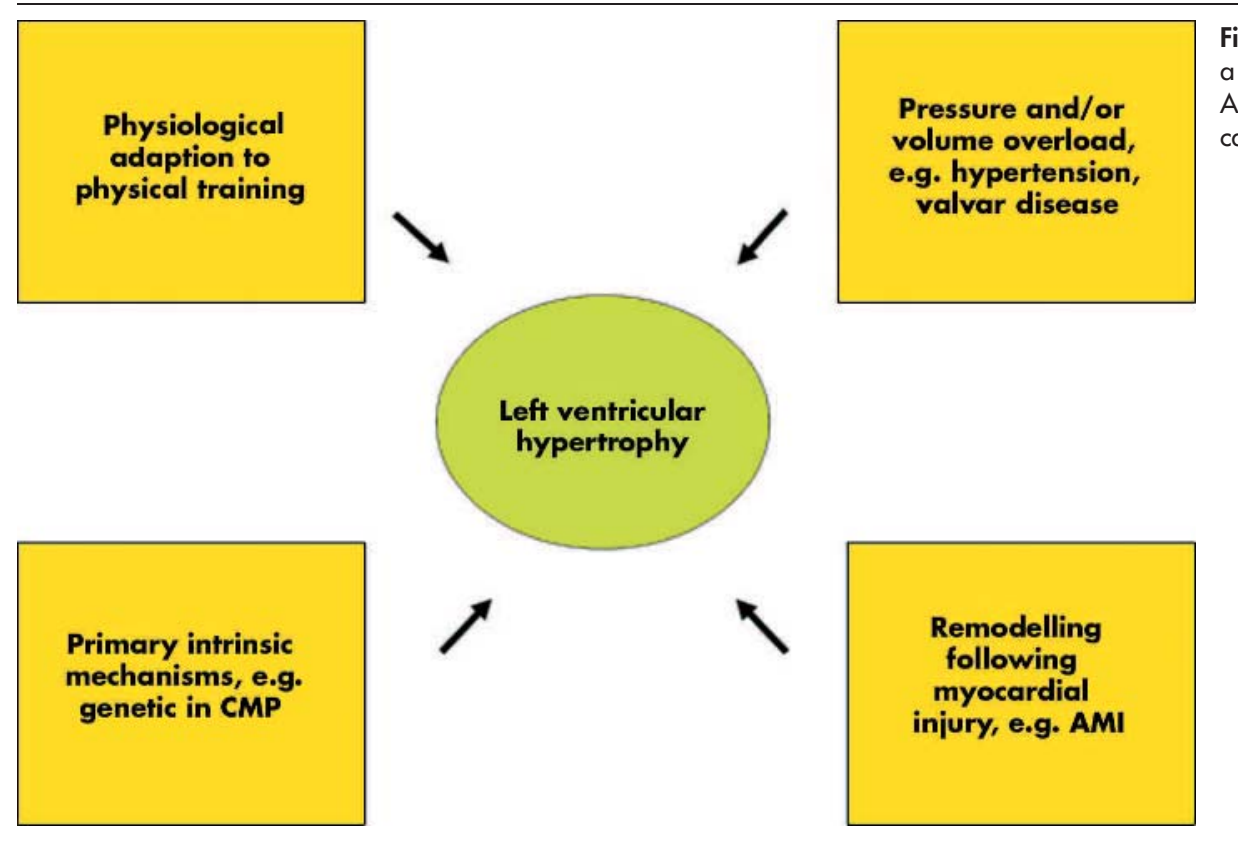

Figure 1 Left ventricular hypertrophy, a condition with variable background. $\mathrm{AMI}$, acute myocardial infarction; CMP, cardiomyopathy.

closely related to LV wall thickness probably reflecting pure pressure load, systolic blood pressure is more closely related to LV mass, suggesting an influence of both pressure and volume load. However, the modest correlation between blood pressure and LV mass suggests additional important factors.

Important non-haemodynamic factors for the development of LV hypertrophy include trophic influence mediated by the sympathetic nervous system and the renin-angiotensinaldosterone (RAA) system. Noradrenaline (norepinephrine) and other substances with $\alpha$ l adrenergic agonist activity have been shown to induce myocyte hypertrophy in vitro and in vivo. Recent results suggest that an increased cardiac sympathetic neurotransmission plays a part in the increase in LV mass in human hypertension. ${ }^{5}$ However, it is likely that direct haemodynamic effects from sympathetic augmentation in hypertension also contribute to the development of LV hypertrophy. ${ }^{\mathrm{w} 7}$ Insulin has trophic effects, and raised levels

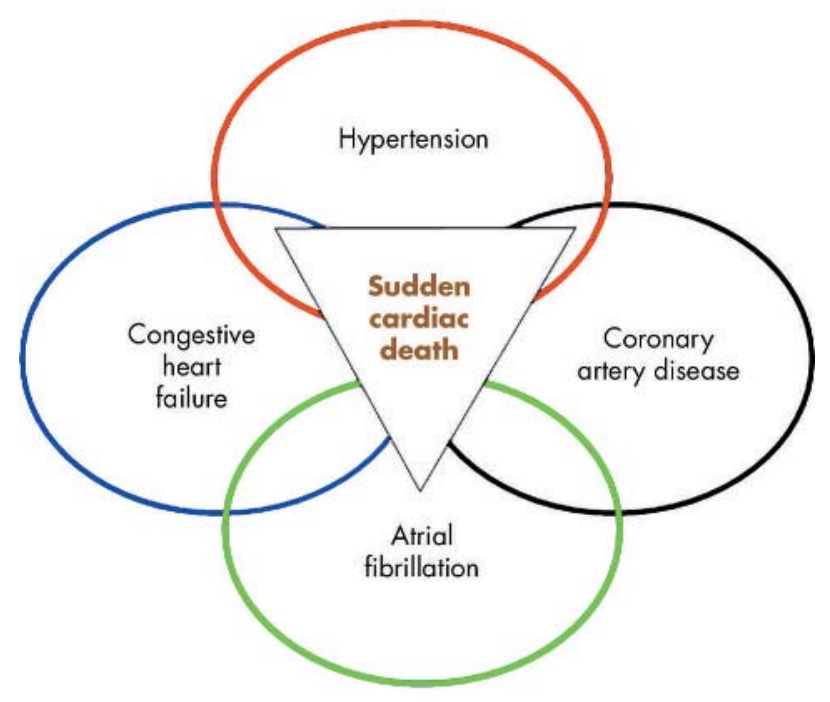

Figure 2 Common and often co-existing cardiovascular conditions with a risk for adverse events including sudden death. of insulin and insulin resistance is more common in patients with cardiovascular disease, including hypertension. Although insulin may have peripheral haemodynamic actions that could increase afterload, it appears that it does not have a direct growth promoting effect on the myocardium. ${ }^{\text {w8 }}$

Circulating plasma concentrations of aldosterone and angiotensin II are related to the extent of LV hypertrophy. Angiotensin II promotes myocyte cell growth, and aldosterone increases the collagen content and stimulates the development of myocardial fibrosis. ${ }^{6}$ Circumstantial evidence suggesting an important role for the RAA system is also the greater reduction in LV mass and of myocardial fibrosis by antihypertensive treatment with drugs that interfere with the actions of the RAA system. ${ }^{7}{ }^{8}$ 9 Furthermore, gene polymorphisms of various components of the RAA system also predict the response in LV mass to antihypertensive treatment. ${ }^{9}$ w10

Demographic determinants such as age, sex, race, and body size also play a role in the development of LV hypertrophy. ${ }^{4}$ Taken together, genetic as well as non-genetic influences on haemodynamic and non-haemodynamic factors seem to cause intracellular stimulation of protein synthesis ultimately influencing the development of LV hypertrophy, as summarised in fig 5 .

\section{DIAGNOSIS OF LV HYPERTROPHY}

Although a physical examination might reveal signs of hypertension and LV hypertrophy by blood pressure measurement, auscultation of an augmented S2 (A2), and palpation of a broad and/or laterally displaced cardiac impulse ("thrusting apex") ECG and echocardiography are the most prominent diagnostic tools. ECG is a readily available and fairly simple diagnostic procedure. An increased load of the left atrium will be reflected in a negative $\mathrm{P}$ wave in the precordial leads. Large QRS amplitudes with deep $\mathrm{S}$ in the anterior leads and high $\mathrm{R}$ in the lateral leads reflect an enlarged LV diameter. A prolonged ventricular activation time-that is, a wide QRS complexas well as ST-T changes reflect altered myocardial depolarisation and repolarisation, respectively. Furthermore, it appears that an anterolateral fascicular block (previously 


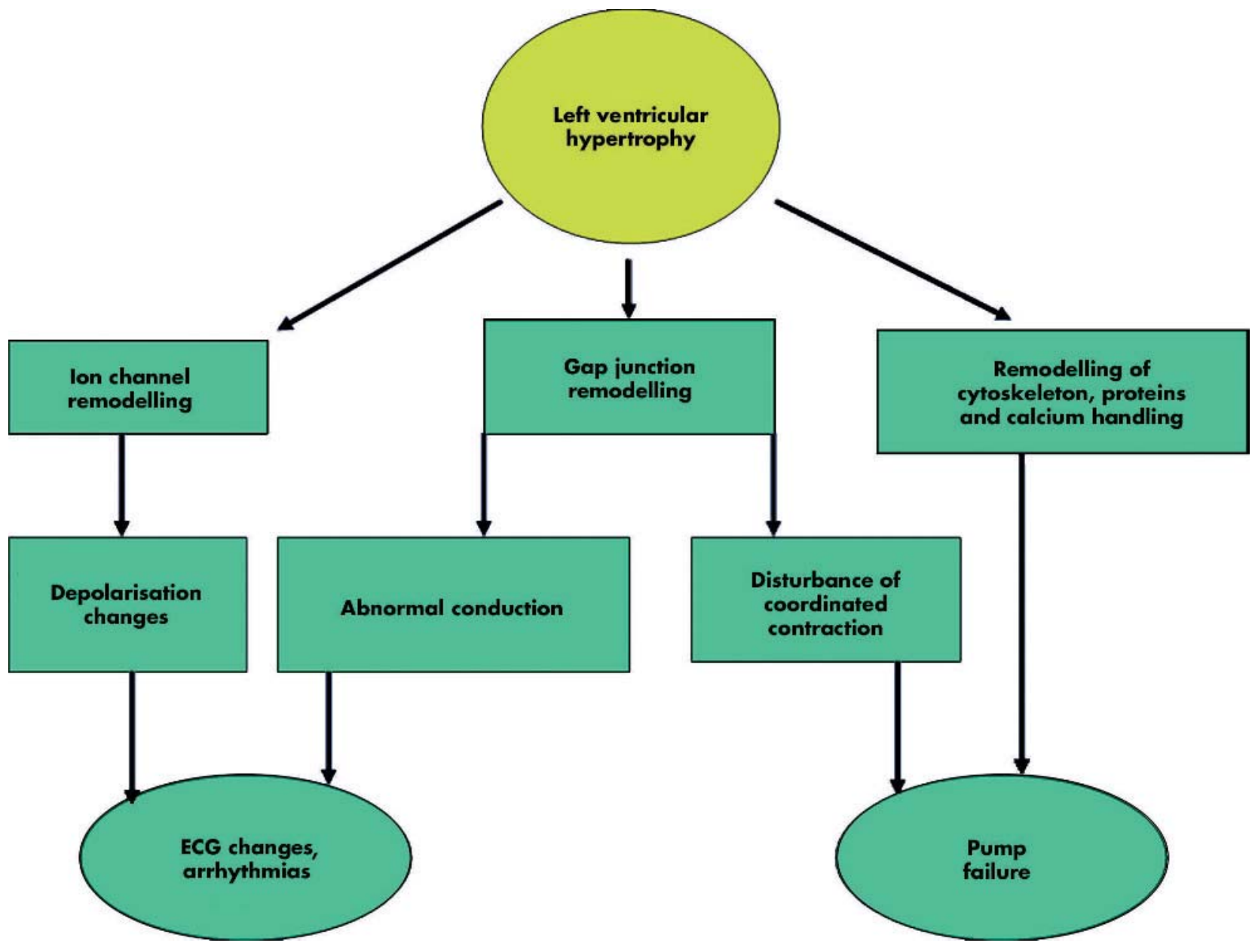

Figure 3 Left ventricular hypertrophy from an electromechanical perspective.

"hemi-block") in hypertensive patients is suggestive of LV hypertrophy. Various combinations of criteria have been suggested to identify LV hypertrophy by the ECG. While the specificity for LV hypertrophy is high ( $90 \%$ or greater), the

\section{LV hypertrophy and hypertension}

Hypertension promotes development of ischaemic injury to myocardium with decreased tolerability to both ischaemia and reperfusion because of:

- Accelerated coronary atherosclerosis causing stenotic lesions

- Increased afterload increasing workload and myocardial oxygen demand

- Factors related to LV hypertrophy

- impaired coronary vasodilator reserve

- increased myocardial cell diameter without proportional proliferation of capillary vessels results in decreased capillary density and increased diffusion distance

- decreased high energy phosphate content

- impaired fatty acid oxidation

- reduced myocardial glucose transport into the cell

As a result there will be an increased ischaemic zone on top of a "reduced repolarisation reserve" and hence increased risk for ventricular fibrillation and sudden cardiac death. sensitivity is low $(20-40 \%)$, although more recent criteria may yield higher diagnostic accuracy. w11 w12

Echocardiography can provide relatively accurate measurements of the intraventricular septum, the posterior LV wall thickness, and the LV diameter in diastole. With the appropriate mathematical formulas these measurements can be used to calculate LV mass. Most commonly this is done according to the Penn convention. ${ }^{\text {wl3 }}$ Because LV mass is related to body size, an indexation of LV mass to body size is important. Most often this is done by dividing with the body surface area. This may, however, misclassify obesity induced LV hypertrophy as normal, and indexing LV mass to its allometric power (that is, LV mass/height ${ }^{2.7} ; \mathrm{g} / \mathrm{m}^{2.7}$ ) would be a preferred approach in this case. The presence of $\mathrm{LV}$ hypertrophy is often considered when LV mass $>116 \mathrm{~g} / \mathrm{m}^{2}$ for men and $>104 \mathrm{~g} / \mathrm{m}^{2}$ for women, ${ }^{w 14}$ or $>125 \mathrm{~g} / \mathrm{m}^{2}$ for men and women, ${ }^{\mathrm{w} 15}$ or $>51 \mathrm{~g} / \mathrm{m}^{2.7}$ for men and women. ${ }^{\mathrm{wl}}$ Other partition values have been proposed, and were generally higher in earlier investigations. ${ }^{\text {w17 }}$

Echocardiographic assessment of LV hypertrophy has a high specificity and sensitivity (both $\geqslant 80 \%$ ). Echocardiography can also provide important additional information, such as other reasons for LV hypertrophy (for example valvar disease, hypertrophic cardiomyopathy), LV geometric pattern, and information on systolic and diastolic function. Recent development in echocardiographic techniques such as 


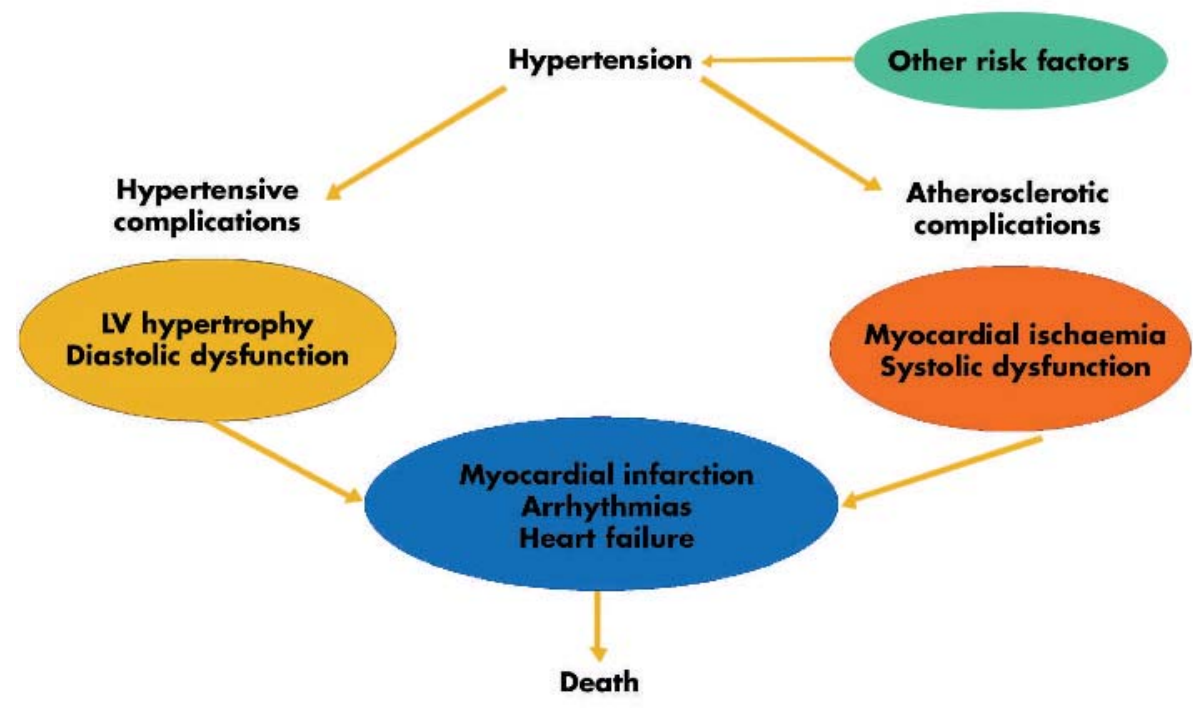

Figure 4 The risk for cardiac events in hypertension. LV, left ventricular.

Adapted from Kahan. ${ }^{4}$

tissue velocity imaging appear to be more sensitive than traditional Doppler echocardiography to detect early signs of hypertensive heart disease such as systolic or diastolic dysfunction. New three dimensional imaging techniques, including magnetic resonance imaging, advanced computed tomography techniques and three dimensional echocardiography, can measure myocardial mass more accurately than conventional echocardiographic techniques. This may offer advantages, especially in relation to mechanistic studies, but may also give additional information on myocardial fibrosis and other structural alterations.

The prevalence of LV hypertrophy increases with the severity of hypertensive disease. By and large, one third to one half of hypertensive patients have LV hypertrophy. Obviously, the partition values used for the definition of an abnormal LV mass and the diagnostic procedures are critical. The actual prevalence of structural changes of the myocardium in hypertension may, however, be higher. ${ }^{\text {w18 }}$
There are four distinct patterns of LV geometry in hypertension based on the relation between the cavity size and the wall thickness-that is, relative wall thickness ${ }^{\text {w19 }}$ (fig 6).

\section{HYPERTROPHY AS A RISK FACTOR}

Myocardial hypertrophy established by ECG or by echocardiography is a strong and independent risk factor for cardiovascular morbidity (including congestive heart failure, coronary heart disease, atrial fibrillation, supraventricular and ventricular arrhythmias, and stroke) and mortality, as well as for all cause mortality in the general population, in hypertensive patients, and in those with coronary artery disease. ${ }^{1}{ }^{\text {w20 }}$ There is a two- to threefold increased risk for having a non-fatal or fatal cardiovascular event in the presence of LV hypertrophy. ${ }^{1}{ }^{\text {w20 }}$ It appears that LV hypertrophy is a stronger risk factor than blood pressure, smoking, or cholesterol, and in multivariate analyses only age and LV

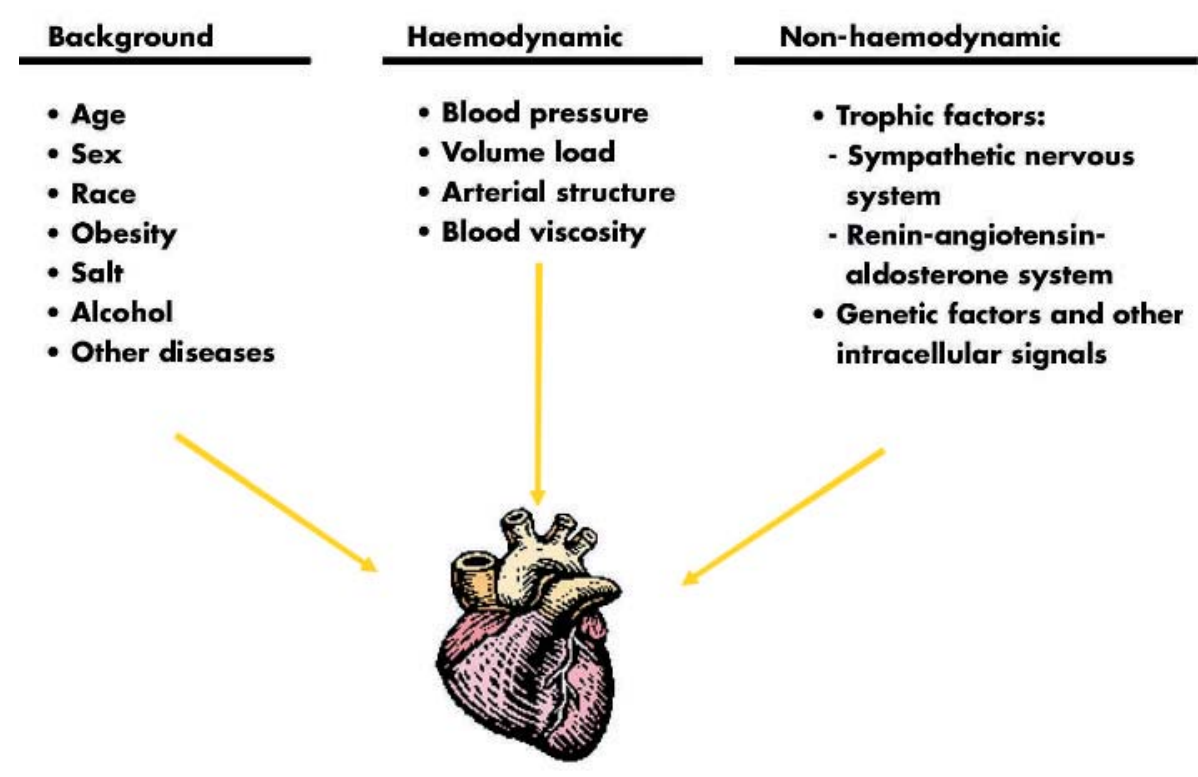

Figure 5 Multiple determinants for left ventricular hypertrophy. Adapted from Kahan. ${ }^{4}$

Trophic factors:

Sympathetic nervous

stem

Renin-angiotensin-

Genetic factors and other

intracellular signals 
Eccentric

Volume overload

Dynamic excercise

254
May be physiological adaption Obesity

Relative increase in myocytes
Concentric

Pressure overload

Static exercise

Pathophysiological condition

Hypertension

Relative increase in connective

tissue and fibrosis
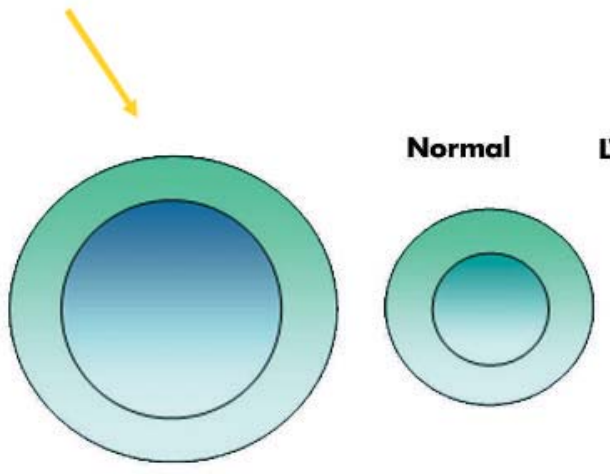

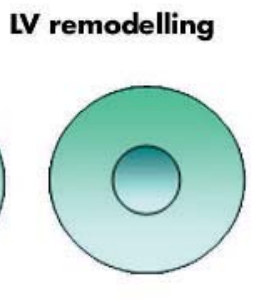

Figure 6 Left ventricular (LV) geometry in hypertension: four patterns with different prognostic implications. hypertrophy remain independent prognostic factors. Of note, the risk for an event is lowest in hypertensive patients with normal LV geometry, followed by those with concentric remodelling, those with eccentric LV hypertrophy, and is highest among those with concentric LV hypertrophy..$^{10}$ Regression of LV hypertrophy by antihypertensive treatment reduces cardiovascular morbidity and mortality and thus improves prognosis. ${ }^{11}$ These results, based on echocardiographic evaluation of LV hypertrophy, are consistent with earlier observations using serial assessment of ECG.

Several mechanisms contribute to the increased risk for a cardiac event in the hypertensive patient. First, the development of LV hypertrophy and myocardial fibrosis leads to LV diastolic dysfunction, an important factor in the evolution of congestive heart failure. Second, hypertension promotes the development of coronary artery atherosclerosis, which increases the risk for an acute myocardial infarction, with subsequent systolic dysfunction and congestive heart failure. Indeed, hypertension remains the most important aetiology for the development of congestive heart failure, followed by ischaemic heart disease. ${ }^{\mathrm{w} 21}{ }^{\mathrm{w} 22}$ In addition, the increase in arterial stiffness and subsequent afterload caused by atherosclerotic disease augments myocardial oxygen demand and can cause myocardial ischaemia. Furthermore, an increased myocardial mass and interstitial myocardial fibrosis is associated with a reduced coronary flow reserve. Consequently, not only is there an increased risk for myocardial ischaemia, but the tolerability is impaired. ${ }^{\text {w23 }}$ w24 Thus, intermittent myocardial ischaemia, with transient diastolic dysfunction, may lead to myocardial scarring and systolic LV dysfunction, and also promote potentially malignant arrhythmias. Importantly there is also an increased risk for atrial fibrillation, an important risk factor for congestive heart failure and thromboembolic cardiovascular complications.

\section{ARRHYTHMOGENESIS IN LV HYPERTROPHY}

A strong chain of evidence for the mechanisms behind ventricular arrhythmia in myocardial hypertrophy has been
LV hypertrophy and hypertension: clinical implications

- In hypertensive patients, evaluate left ventricular geometry and function by echocardiography

- Recognise hypertension induced left ventricular hypertrophy as a latent long QT syndrome

- Treat high blood pressure and reverse hypertrophy

- Avoid hypokalaemia, bradycardia, and QT prolonging drugs - that is, anything that prolongs repolarisation time

established. There is also a strong link between ventricular arrhythmias and congestive heart failure. ${ }^{3}{ }^{\mathrm{w} 2}$ Thus, a relation between prolongation and heterogeneity (dispersion) of repolarisation and polymorphic ventricular arrhythmia, especially torsade de pointe ventricular tachycardia, has been established, ${ }^{312}{ }^{13}$ as outlined below.

Prolonged action potential duration is a salient feature in myocardial hypertrophy, independent of cause. This may lead to early after depolarisations and triggered activity, and in the intact heart increased dispersion of repolarisation may sustain the arrhythmia. In the hypertrophic myocardium myofibrillar disarray, heterogeneous gap junction distribution, and fibrosis are additional potentially arrhythmogenic components.

Torsades de pointes ventricular arrhythmias are typically rate dependent, following sequences of short-long RR intervals. Consistent with these observations is the steeper relation between the QT and RR intervals (QT/RR relation) induced by drugs known to be proarrhythmic. ${ }^{\text {w25 }}{ }^{\text {w26 }}$ This phenomenon, referred to as reverse use dependence, is also present in LV hypertrophy. ${ }^{\mathrm{w} 27}$ Of interest is that this pattern is typical also in the congenital LQTS, ${ }^{\text {w28 }}$ and was recently found to be predictive of sudden death in post-myocardial infarction patients. ${ }^{\text {w29 }}$ This suggests electrophysiological similarities between hypertensive LV hypertrophy and other conditions associated with proarrhythmic potential, such as 
the LQTS, all of which are related to polymorphic ventricular tachycardia.

The repolarisation time and its dispersion are related to the degree of LV hypertrophy. Antihypertensive treatment reduces LV mass. ${ }^{\text {w30-32 }}$ Furthermore, it reduces both repolarisation time and its dispersion, ${ }^{14-17}$ w33 the incidence and severity of ventricular arrhythmia, ${ }^{18}$ and the risk for cardiovascular events. ${ }^{11} 19 \mathrm{w} 34$ As discussed, the RAA system plays an important role for the control of LV mass and structure. Consequently, recent interest has focused on angiotensin II type 1 receptor blockade (ARB), because this class of drugs might interact with several possible arrhythmogenic mechanistic factors induced by angiotensin II acting on its principal receptor, the type 1 receptor (fig 7).

Animal studies have shown important arrhythmogenic effects of angiotensin II on the cardiac conduction system, which has a high density of angiotensin II type 1 receptors. Angiotensin II reduces intracellular resistance, decreases conduction velocity, and shortens the refractory period in cardiac myocytes. ${ }^{\mathrm{w} 35 \mathrm{w} 36}$ Local application of angiotensin II to the heart causes increased dispersion of the action potential durations. ${ }^{\text {w36 }}$ In addition, it has been shown in a rat model of eccentric LV hypertrophy induced by aortic banding, that ARB prevents the development of disorganised cell-to-cell connections, as assessed by the distribution of gap junction protein Cx $43 .{ }^{\text {w37 }}$ Angiotensin II can facilitate noradrenaline release by activation of prejunctional angiotensin II type 1 receptors. ${ }^{\text {w38 }}$ Abnormal myocardial fibrosis is a common finding in LV hypertrophy, ${ }^{\mathrm{w} 39}$ but is reduced by drugs that block the actions of the RAA system, ${ }^{11}{ }^{140}$ which thereby may reduce the risk for malignant arrhythmia and sudden death.
Also, regression of structural vascular changes that causes a reduced coronary flow reserve ${ }^{\mathrm{w} 41}$ could probably contribute to reduce the risk of arrhythmias. Finally, animal data provide intriguing results, since not only stretch induced by increased afterload but also stretch induced by sudden increases in preload might provoke arrhythmias. ${ }^{\mathrm{w} 42}$ This is of potential interest in the clinical setting of hypertension and eccentric LV hypertrophy, in which an increase in preload often is present.

\section{CLINICAL IMPLICATIONS}

Torsades de pointes ventricular tachycardias are uncommon both in the congenital and acquired forms of LQTS. Furthermore, these arrhythmias are potentially life threatening, and difficult to predict on the individual level, although risk factors have been identified. Therefore prevention is of utmost importance, which requires knowledge about risk conditions-that is, about conditions with a "reduced repolarisation reserve" -and about factors influencing ventricular repolarisation. Anything that might add risk, such as hypokalaemia and bradycardia, should be avoided. Furthermore, because the arrhythmia risk is so closely related to structure, imaging techniques should be applied for proper identification of risk individuals, and treatment that can reverse myocardial hypertrophy should be applied. New drug classes interacting with the RAA system such as angiotensin converting enzyme (ACE) inhibitors, ARBs, and aldosterone receptor antagonists could be the beginning of a new era of antiarrhythmic drug treatment. We may see the focus shift from the more secondary electrophysiological aspects and their treatment to the arrhythmia substrate itself and to

\section{Increased wall stress and stretch}

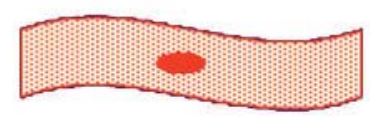

\section{Facilitates local NA release and inhibits central vagal activity}

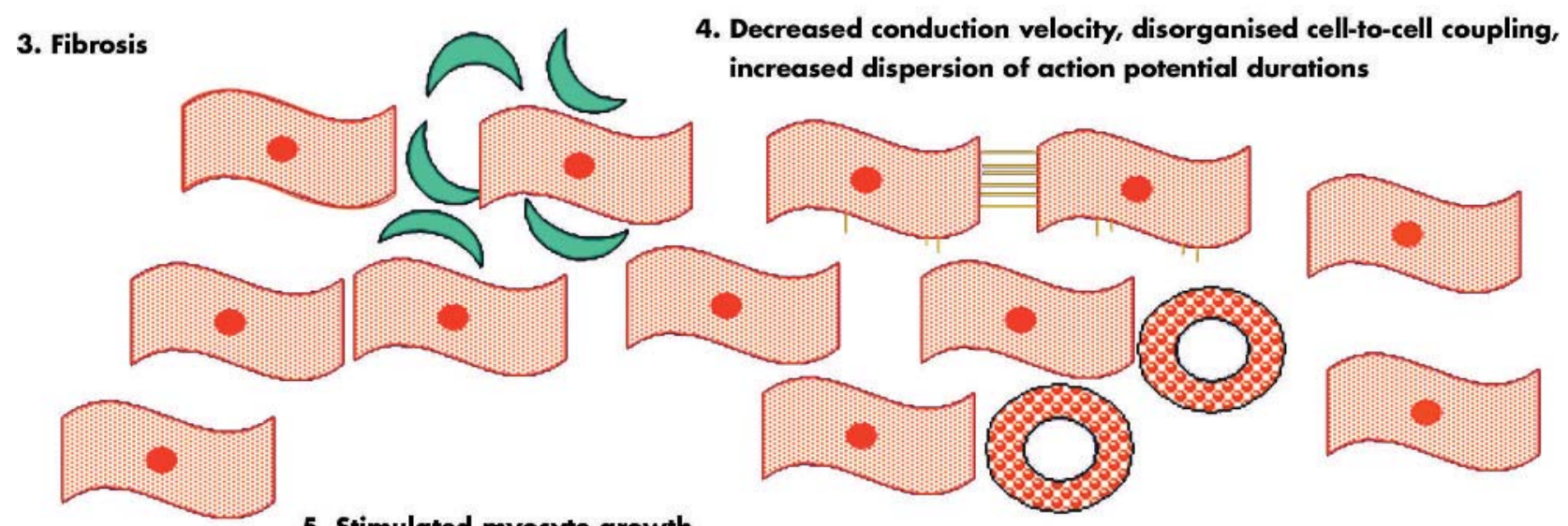

5. Stimulated myocyte growth

\section{Structural vascular changes}

Figure 7 Some angiotensin II related effects of relevance for arrhythmogenesis. See text for details and references. NA, noradrenaline/ norepinephrine. 
reversing structural remodelling-similar to what has been suggested for the treatment of atrial fibrillation, for example. $^{20}$ w43

\section{Authors' affiliations}

256 Hospital, Stockholm, Sweden

L Bergfeldt, Sahlgrenska Academy, University of Gothenburg,

Sahlgrenska University Hospital/S, Gothenburg, Sweden

\section{REFERENCES}

1 Vakili BA, Okin PM, Devereux RB. Prognostic implications of left ventricular hypertrophy. Am Heart J 2001;141:334-41.

- A recent meta-analysis of the prognostic implications of LV mass.

2 Lips DJ, deWindt $\sqcup$, van Kraaii DJW, et al. Molecular determinants of myocardial hypertrophy and failure: alternative pathways for beneficial and maladaptive hypertrophy. Eur Heart J 2003;24:883-96.

- A recent review of the molecular background to left ventricular hypertrophy with a haemodynamic focus.

3 Pacifico A, Henry PD. Structural pathways and prevention of heart failure and sudden death. J Cardiovasc Electrophysiol 2003;14:764-75.

- Myocardial disease associated with heart failure and sudden cardiac death reviewed from a macroscopic and microscopic anatomy perspective with a focus on the prevention of sudden cardiac death.

4 Kahan T. The importance of left ventricular hypertrophy in human hypertension. J Hypertens 1998;16(suppl 7):S23-9.

5 Schlaich MP, Kaye DM, Lambert E, et al. Relation between cardiac sympathetic activity and hypertensive left ventricular hypertrophy. Circulation 2003;108:560-5

6 Weber KT, Brilla CG, Campbell SE, et al. Myocardial fibrosis: role of angiotensin II and aldosterone. Basic Res Cardiol 1993;88:107-24.

- Excellent overview of the importance of the renin-angiotensinaldosterone system in the development of myocardial hypertrophy and fibrosis.

7 Klingbeil AU, Schneider M, Martus $P$, et al. A meta-analysis of the effects of treatment on left ventricular mass in essential hypertension. Am J Med 2003;115:41-6.

8 Brilla CG, Funck RC, Rupp H. Lisinopril-mediated regression of myocardial fibrosis in patients with hypertensive heart disease. Circulation 2000;102:1388-93.

- This study shows that treatment with an ACE inhibitor reduces myocardial fibrosis, evaluated by endomyocardial biopsies, and improves diastolic function. These effects go beyond the effects of blood pressure reduction alone.
9 Kurland L, Melhus $\mathrm{H}$, Karlsson J, et al. Polymorphisms in the angiotensinogen and AT1-receptor gene are related to change in left ventricular mass during antihypertensive treatment. J Hypertens 2002;20:657-63.

10 Koren MJ, Deveraux RB, Casale PN, et al. Relation of left ventricular mass and geometry to morbidity and mortality in uncomplicated essential hypertension. Ann Intern Med 1991;114:345-52.

- Seminal work on the prognostic implications of different left ventricular geometries in hypertensive patients.

11 Verdecchia P, Angeli F, Borgioni C, et al. Changes in cardiovascular risk by reduction of left ventricular mass in hypertension: a meta-analysis. Am J Hypertens 2003;11:895-9.

- A recent meta-analysis on the prognostic implications of changes in LV mass by antihypertensive treatment.

12 Aronson R. Mechanisms of arrhythmias in ventricular hypertrophy. J Cardiovasc Electrophysiol $1991 ; 2: 249-61$.

- References 12 and 13 review important in vitro and in vivo studies.

13 Hart G. Cellular electrophysiology in cardiac hypertrophy and failure. Cardiovasc Res 1994;28:933-46.

14 Gonzalez-Juanatey JR, Garcia-Acuna JM, Pose A, et al. Reduction of QT and QTc dispersion during long-term treatment of systemic hypertension with enalapril. Am J Cardiol 1998;81:170-4.

15 Karpanou EA, Vyssoulis GP, Psichogios A, et al. Regression of left ventricular hypertrophy results in improvement of QT dispersion in patients with hypertension. Am Heart J 1998;136:765-8.

16 Lim PO, Nys M, Naas AA, et al. Irbesartan reduces QT dispersion in hypertensive individuals. Hypertension 1999;33:713-8.

17 Malmqvist K, Kahan T, Edner M, et al. Comparison of actions of irbesartan versus atenolol on cardiac repolarization in hypertensive left ventricular hypertrophy: results from the Swedish irbesartan left ventricular hypertrophy investigation versus atenolol (SILVHIA). Am J Cardiol 2002;90:1107-12.

18 Messerli FH. Hypertension and sudden cardiac death. Am J Hypertens 1999;12:181S-8S

19 Lindholm LH, Dahlöf B, Edelman JE, et al. Effect of losartan on sudden cardiac death in people with diabetes: data from the LIFE study. Lancet 2003:362:619-20.

20 Members of the Sicilian Gambit. New approaches to antiarrhythmic therapy. Emerging therapeutic applications of the cell biology of cardiac arrhythmias. Eur Heart J 2001 ;22:2148-63.

- An electrophysiological approach to antiarrhythmic treatment describing the arrhythmogenic myocardial substrate, genetic factors, and environmental stresses, and principles of rational drug design.

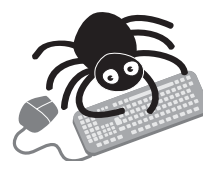

Additional references appear on the Heart websitehttp://www.heartinl.com/supplemental 